\title{
Dimensional crossover in alternating spin chains
}

\author{
Adolfo E. Trumper ${ }^{1}$ and Claudio J. Gazza \\ Instituto de Física Rosario (CONICET) and Universidad Nacional de Rosario, \\ Boulevard. 27 de febrero 210 bis,(2000) Rosario, Argentina.
}

\begin{abstract}
The effect of antiferromagnetic interchain coupling in alternating spin $(1,1 / 2)$ chains is studied by mean of spin wave theory and density matrix renormalization group(DMRG). Two limiting cases are investigated, the two-leg ladder and its two dimensional (2D) generalization. For the 2D case, spin wave approximation predicts a smooth dimensional crossover keeping the ground state ordered, whereas in the ladder case the DMRG results show a gapped ground state for any $J_{\perp}>0$. Furthermore, the behavior of the correlation functions closely resemble the uniform spin-1/2 ladder. However, for small $J_{\perp}$, the gap behaves quadratically as $\Delta \sim 0.6 J_{\perp}^{2}$. Similarly to uniform spin chains, it is conjectured an analogous spin gap behavior for an arbitrary number of mixed spin chains.
\end{abstract}

Key words: Mixed spin-chains, Antiferromagnetism, Quasi-onedimensional systems.

In the last two decades, quantum magnetism in low dimensions has become a main topic in condensed matter physics. Among the several systems studied in this area, quasi-onedimensional magnets with mixed spin composition are of special interest since their recent synthesization[1,2]. In particular, such ferrimagnetic compounds are all composed by weakly coupled alternating spin chains (Fig. 1(b) represents the ladder case of such topology). There is another family of compounds like $\mathrm{MnCu}(\mathrm{pba})\left(\mathrm{H}_{2} \mathrm{O}\right)_{3} \cdot 2 \mathrm{H}_{2} \mathrm{O}$, with $\mathrm{pba}=1,3-$ propylenebis(oxamato), where the ground state is a non-magnetic one, although it is composed of weakly coupled alternating spin $\left(\mathrm{Mn}^{I I} \mathrm{Cu}^{I I}\right)$ chains (Fig. 1(a)). This last topology, motivated us to study the ground state properties of an al-

1 Corresponding author. Fax: +341-54-4821772

E-mail: trumper@ifir.edu.ar, E-mail: gazza@ifir.edu.ar (a)

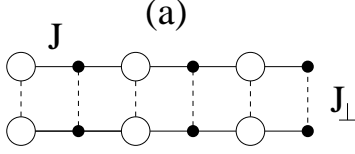

$\mathrm{S}=1$ (b)

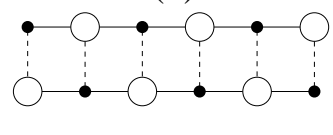

- $\mathrm{s}=1 / 2$

Fig. 1. Two alternating spin systems: (a) has a singlet ground state, (b) has a ferrimagnetic one.

ternating spin ladder (ASL) of type (a) and its 2D generalization with the following Hamiltonian,

$$
H=J \sum_{\langle i, j\rangle_{\|}} \mathbf{S}_{\mathbf{i}} \cdot \mathbf{S}_{\mathbf{j}}+J_{\perp} \sum_{\langle i, j\rangle_{\perp}}\left(\mathbf{S}_{\mathbf{i}} \cdot \mathbf{S}_{\mathbf{j}}+\mathbf{s}_{\mathbf{i}} \cdot \mathbf{S}_{\mathbf{j}}\right),
$$

where $\mathbf{S}_{\mathbf{i}}\left(\mathbf{s}_{\mathbf{i}}\right)$ represents a spin-1 (spin-1/2), $\langle i, j\rangle_{\|}$ $\left(\langle i, j\rangle_{\perp}\right)$ denotes nearest neighbors along horizontal (vertical) direction, with $J$ and $J_{\perp}$ defined positive.

Recently, it was shown how the spin wave (SW) series converges correctly to the DMRG results 
Table 1

Spin wave prediction for the relative magnetizations vs. $J_{\perp}$ for the 2D case.

\begin{tabular}{lllllll}
\hline$J_{\perp}$ & 0 & 0.2 & 0.4 & 0.6 & 0.8 & 1.0 \\
\hline$m_{1} / S$ & 0.695 & 0.721 & 0.739 & 0.746 & 0.738 & 0.733 \\
\hline$m_{2} / s$ & 0.390 & 0.597 & 0.644 & 0.712 & 0.730 & 0.739 \\
\hline
\end{tabular}

in ferrimagnetic chains[3]. Hence, SW seems to be a good starting point technique to study the crossover from $1 \mathrm{D}$ to $2 \mathrm{D}$, where $\mathrm{SW}$ is even more reliable. In table 1 we show the relative values of the magnetizations $m_{1} / S$ and $m_{2} / s$, for the spin1 and spin-1/2, respectively, as a function of $J_{\perp}$. It can be noticed how the relative values tend to a similar value around 0.73 as the isotropic limit is reached. In this case we obtained a value $m_{2} / s=$ 0.739 bigger than the uniform spin- $1 / 2$ case $\mathrm{m} / \mathrm{s}=$ $0.606[4]$. Using mean-field arguments, such a robust magnetization is due to the more stronger field caused by the spin- 1 neighbors. As general result we observed that the effect of $2 \mathrm{D}$ coupling is the enhancement of the magnetizations, being the crossover completely smooth. On the other hand, for the ladder case, Fig. 1(a), it can be proved that quantum fluctuations prevent the ordering. This feature was previously pointed out by Fukui and Kawakami[5] and, in agreement with our DMRG findings, suggests the opening of a spin gap that is impossible to predict within a SW framework.

For the two-leg ASL we used the DMRG method[6], using both, the finite and infinite algorithm, with open boundary conditions to study the spin gap and the correlations functions respectively with $J_{\perp}$. Most of the calculations was carried out keeping between 200 and 400 density matrix states, with a truncation error $O\left(10^{-8}\right)$ at worst and $O\left(10^{-12}\right)$ in the best case[7]. In Fig.2 we show the gap, that for small values of $J_{\perp}$ (below 0.3 ) behaves quadratically as $\Delta \sim 0.6 J_{\perp}^{2}$ and for bigger values of $J_{\perp}$ it turns out quite linear corresponding to the strong coupling regime. For $J_{\perp}=1$ we obtained $\Delta=0.334$. Consistently with these features, when extrapolating the gap for different $J_{\perp}$, we distinguished two scaling regimes at $J_{\perp} \sim 0.3$ as it is shown in the inset of Fig.2. It is

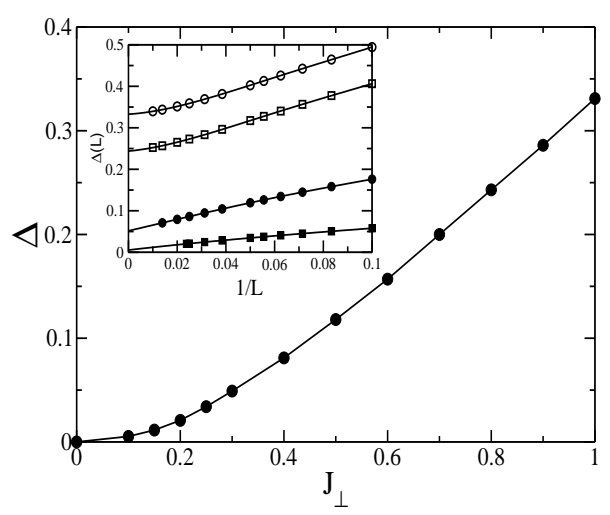

Fig. 2. Spin-gap versus $J_{\perp}$ obtained with DMRG. Inset: Spin gaps as a function of $1 / L$ for $J_{\perp}=1$ (empty circle), 0.8 (empty square), 0.3 (filled circle), and 0.1 (filled square). Solid lines are polynomial fits of the form[12] $\Delta(L)=\Delta+a_{1} / L+a_{2} / L^{2}+a_{3} / L^{3}+\ldots$ where, $\Delta(L)=E\left(L, S_{z}=1\right)-E\left(L, S_{z}=0\right)$ with $E\left(L, S_{z}\right)$ the ground state energy for a "chain" with $L$ rungs (ranging from 10 to 100), and $z$ component of total spin $S_{z}$.

interesting to compare our results for the weakly coupled regime with that of the uniform spin-1/2 ladder (USL) that are known to behave linearly as $\Delta \sim 0.41 J_{\perp}[8,9]$. We think that this discrepancy reflects the quite distinct underlying physics behind the decoupled regime. In order to get a deeper insight of the ground state configuration of the ASL we computed the local bond strengths and compared them with the USL. In Fig.3(a), we present the normalized values of $\left\langle\mathbf{S}_{i} \cdot \mathbf{s}_{i+1}\right\rangle$ along the leg. A similar monotonic behavior with $J_{\perp}$ is observed for ASL and USL. In Fig.3(b) we show the normalized values of $\left\langle\mathbf{S}_{i} . \mathbf{S}_{i+1}\right\rangle$ and $\left\langle\mathbf{s}_{i} \cdot \mathbf{s}_{i+1}\right\rangle$, corresponding to the vertical spin-1 and spin-1/2 rungs, respectively. It can be noticed that the spin-1 rungs remain always larger than the spin$1 / 2$ ones in the ASL. This is non-trivial at all since the quantities are normalized to its free bond case. In the strong coupling regime the ground state is composed by an alternated collection of spin- 1 and spin- $1 / 2$ singlets. As $J_{\perp}$ is decreased, each kind of spin will resonate among its different neighbors reducing their correlation along the rungs. Nevertheless, from the valence bond solid picture, it is easy to realize (see Fig.4), that the reduction of the correlation in the spin- 1 rung will 


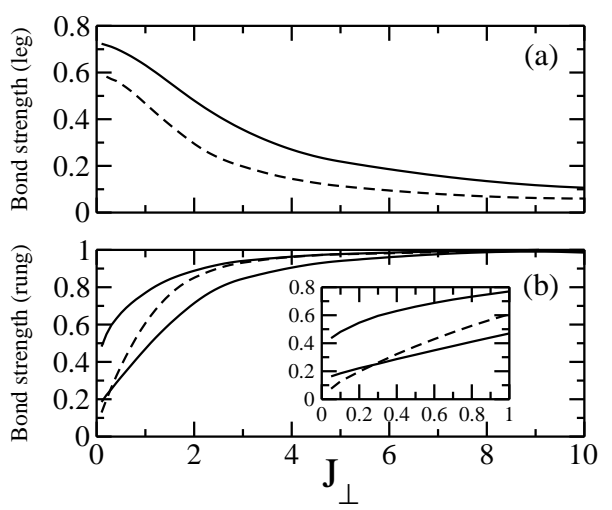

Fig. 3. Normalized bond strength as a function of $J_{\perp}$. (a) $\left\langle\mathbf{S}_{i} \cdot \mathbf{s}_{i+1}\right\rangle / A$ along the leg for ASL (solid line) and $\left\langle\mathbf{s}_{i} . \mathbf{s}_{i+1}\right\rangle / B$ for USL (dashed line), and (b) The upper solid line is for spin-1 rungs $\left\langle\mathbf{S}_{i} . \mathbf{S}_{i+1}\right\rangle / C$ while the lower solid line is for spin- $1 / 2$ rungs $\left\langle\mathbf{s}_{i} \cdot \mathbf{s}_{i+1}\right\rangle / D$. The constants $A=-1, B=-3 / 4$, and $C=-2$ are the corresponding free bond cases. The dashed line is the USL case. In the inset, the region where the crossing occurs has been amplified.

be less important than in the spin- $1 / 2$ rung. It is also remarkable the crossing between the spin$1 / 2$ rungs values for ASL and USL that occurs at $J_{\perp} \sim 0.3$. Even if we have not a physical explanation for that we believe that our results for the gap and the local bond strengths are connected and indicate a change of regime for the above value of $J_{\perp}$. In addition we studied the spin cor-

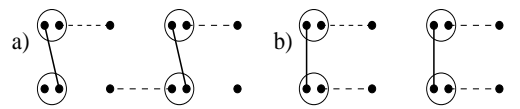

Fig. 4. (a) and (b) represent two possible configurations among which the ground state resonates once $J_{\perp}$ is decreased coming from strong coupling.

relations functions along the legs[7]. We observed that the correlation functions along the leg decay exponentially for the whole range of interchain coupling, with $\xi^{A S L} \rightarrow \infty$ in the limit $J_{\perp} \rightarrow 0$, like in USL[8]. When $J_{\perp}$ is strictly zero there is a transition to two ferrimagnetically ordered chains in the ASL. Using a semi-log plot of such correlations, we estimated the correlation length for $J_{\perp}=0.1$ being $\xi^{A S L} \sim 30$, and $\xi^{U S L} \sim 25$ lattice spacings. On the other hand, for $J_{\perp}=1$ we estimated $\xi^{A S L} \sim 5$, whereas in the USL it was found $\xi^{U S L} \sim 3[8,12]$. Except for the differences found in the weakly coupled regime, our DMRG results suggest a strong similarity between ASL and USL.

Finally, from the two limiting cases we have studied, it is possible to argue a more general statement about the behavior of the spin gap for an arbitrary number of coupled mixed spin chains. First, we can rigorously say that any odd number of chains have always a ferrimagnetic ground state, due to Lieb-Mattis theorem[10], so, it will be gapless -but ordered-. Then, complementing our results with the conjecture that an even number of chains will be gapped and that similarly to uniform ladders[11-13] this gap will decrease to zero in the 2D limit, it is recovered an analogous spin gap behavior to the uniform spin-1/2 case. However, for a finite odd number of chains, these gapless states have a completely different nature.

This work was done under PICT grant N0303833 (ANPCYT). The authors acknowledge partial financial support from Fundación Antorchas.

\section{References}

[1] Y. Pei, M. Verdaguer, O. Kahm, J. Sletten and J. P. Renard, Inorg. Chem.26, 138 (1987).

[2] M. Hagiwara, K. Minami, Y. Narumi, K. Tatani, and K. Kindo, J. Phys. Soc. Jpn. 67, 2209 (1998).

[3] N. B. Ivanov Phys. Rev. B 57, R14024 (1998); N. B. Ivanov, Phys. Rev. B 62, 3271 (2000).

[4] P.W. Anderson, Phys. Rev. 86, 694 (1952).

[5] T. Fukui and N. Kawakami, Phys. Rev. B 57, 398 (1998).

[6] S. R. White Phys. Rev. B 48, 10345 (1993).

[7] A. E. Trumper and C. Gazza, to be published in Phys. Rev. B.

[8] M. Greven, R. J. Birgeneau, and U. J. Wiese, Phys. Rev. Lett. 77, 1865 (1996).

[9] T. Barnes, E. Dagotto, J. Riera and E. S. Swanson, Phys. Rev. B 47, 3196 (1993).

[10] E. Lieb and D. Mattis, J. Math. Phys.3, 749 (1962).

[11] E. Dagotto and T. M. Rice, Science 271, 618 (1996).

[12] S. R. White, R. M. Noack and D. J. Scalapino. Phys. Rev. Lett. 73, 886 (1994).

[13] T. M. Rice, S. Gopalan and M. Sigrist, Europhys. Lett. 23, 445 (1993); B. Frischmuth, B. Ammon and M. Troyer, PRB 54, R3714 (1996). 